\title{
Dynamic Contextual Usage Metadata for Learning Resource Reuse in Adaptive Environments
}

\author{
Eddie Walsh, Rachael Rafter, Owen Conlan and Vincent Wade \\ Knowledge and Data Engineering Group \\ School of Computer Science and Statistics, \\ Trinity College Dublin, Ireland. \\ \{walshe8,rachael.rafter,owen.conlan,vincent.wade\}@scss.tcd.ie
}

\begin{abstract}
Drawing on the experiences and context of others who have already used a particular resource can greatly facilitate that resource's reuse. Such reuse is essential when the resources in question are digital learning assets, services and models which are expensive both in terms of time and monetary expenditure to develop and use. When these resources are deployed in personalised settings, where each user may be delivered a tailored sequence of resources that uniquely suits their particular needs, gathering and federating a rich view of how these resources are being used becomes important. In this article we describe an approach to facilitating the federating of contextual usage data, which is compiled over the lifecycle of a resource. Given that this data is likely to come from a range of different sources, our approach will need to be able to cope with the high level of heterogeneity expected in terms of its structure, syntax and semantics. We describe how such data may be used to support users in assessing the value of learning resources and facilitating their appropriate reuse.
\end{abstract}

Keywords: Learning Resources, Adaptivity, Usage Metadata, Reuse, Integration

\section{Introduction}

Deciding when and where to appropriately reuse learning resources is far from trivial. Factors such as the domain knowledge, pedagogical soundness and the individuality of users are well documented important considerations for course design [4]. To date, most learning resource metadata has remained rather static, typically summarising the characteristics of the learning resource and the original author's vision of its usage. Dynamic contextual information about how, why and by whom the resource is used is typically not considered. It is this information that is cru- cial for the reuse of learning resources; it informs us about the suitability of the resource for a particular context, and chronicles how the resource is used in practice, which does not necessarily coincide with the original author's expectation of its usage. In short, this data gives course authors access to a rich body of experience that scaffolds their design choices and encourages them to reuse existing learning resources whose appropriateness has been validated via historical evidence.

In this paper we argue that, what we term contextual usage metadata, should supplement the standard metadata associated with learning resources, and importantly, detail when and where the deployment of a resource has been particularly successful. Drawing on the experiences and context of others who have already used a particular resource can greatly facilitate that resource's reuse. Such reuse is essential when the resources in question are digital learning objects, services and models that are costly in terms of time and monetary expenditure to develop and deploy.

Online courses have evolved from sequences of static content into immersive experiences involving both content and services (e.g., voting, rating, blogging) where the students interact, participate and collaborate as they learn. At the same time, online learning resources are serving a more diverse set of learners who may be involved in structured, semi-structured or fully independent learning in both formal and informal settings. Furthermore, personalisation has become a key trend in successful e-learning systems [14]. Previously, such systems followed a "one size fits all" approach, in which learners were presented with the same set of content resources regardless of their individual needs. State-of-the-art e-learning systems today instead deliver tailored content and services to learners, which are uniquely suited to their personal preferences, knowledge, goals and learning style [12]. Accordingly, each individual learning resource can be deployed in a variety of contexts, where it is adapted each time to suit a different set of constraints. 
To provide reuse of learning resources in these environments requires dynamic federation of contextual usage metadata. Various implicit and explicit usage data is generated by multiple, often interchangeable, services and as a result can vary in structural, syntactic and semantic heterogeneity. To achieve federation requires methods to mediate the heterogeneity inherent in various learning environments and their resulting metadata models.

Our contributions in this paper are two-fold. We begin by discussing the federation of contextual usage metadata from multiple heterogeneous sources, and borrow from our previous work on federating user information [15]. Specifically we describe a new approach based on FUMES; an interoperability system for the automatic, on-demand, exchange of user information between multiple heterogeneous sources. Then we move on to describe how federated contextual usage metadata may be used to better facilitate the reuse of learning resources.

In Section 2 we review related work. Section 3 describes our proposed approach for the federation of contextual usage metadata from different heterogenous sources, and Section 4 discusses how such metadata may be exploited to support the reuse of learning resources.

\section{Related Work}

\subsection{Contextual Usage Metadata}

To date in personalised e-learning systems, the metadata used to describe learning resources has remained rather static, and the pool of content and services, as well as the context in which the personalisation is carried out, is very limited [2, 5, 3]. However we are beginning to see an emerging shift towards more openly described resources. For example, services are playing an increasingly pivotal role in modern e-learning systems, facilitating interactions between learner and system. Services by their very nature are typically designed to be open and reusable. Furthermore, e-learning resources are being deployed in more diverse scenarios, that cater not only for the standard classroom style of course, but for independent or collaborating learners as well.

There have however been a few attempts to move towards more dynamic and contextual usage metadata. Contextualised attention metadata $[10,11,16]$ is one example that uses the AttentionXML standard developed in 2004. The attention that a user gives to different resources (such as feeds, blogs, wikis) and any associated interactions are tracked and analysed with respect to the context in which they are carried out. The contextualised attention metadata schema not only records an action that a user took, but also registers when the action took place, through which tool the action took place, what other things the user was doing at the time, the community to which the user belongs, and the user's profile [11]. This can be used to update a user model and provide information about a user's current interests; to provide empirical analysis of the actual use of learning objects to administrators of learning management systems; or finally as input to a recommender system tasked with suggesting new objects to learners, [10].

With similar motivations McCalla describes the ecological approach to designing e-learning environments, in which information about how learning objects are used in practice is accumulated $[8,1]$. The learner model is split into two, where one half represents the long-term characteristics of the learner, and the other represents episodic shortterm information about the learner's experiences with the current learning object. The former might contain information like the preferred learning style, past knowledge and learning goal for example, while the latter would provide traces of the learner's interaction with the learning object, how he evaluated it, and any of his test results relating to the learning object's content.

When the learner consumes a learning object, the current state of his profile (containing both parts) is attached to the learning object. Over time the learning object collects many learner model snapshots which can be mined for usage patterns. The patterns might then be used to inform a recommender system, to determine the strength or weakness of a learning object for a particular pedagogical goal (either for an individual or type of user) or to identify learners who would benefit from working together for example, (the interested reader should refer to [1] for a complete list of intended purposes).

Lehmann et al. [7] refer to usage information about learning resources as lifecycle information. The lifecycle of a learning resource is comprised of a number of phases: authoring (the learning resource is created), provision (it is provided to educators or learners), learning (the resource is used) and re-authoring (the resource is adapted for a new context). In particular when a resource is re-authored, it may be unitised, adapted, updated or re-aggregated as parts are added, adapted, removed, or combined with parts of other learning resources. Like contextualised attention metadata and the data captured by the ecological approach, contextual information is captured about the usage of learning resources. However additionally, so-called relation information is collected when a learning resource is reauthored, which defines the relationship that the resource has with other resources. For example, it is clearly related to its previous version, and it may also be related to other resources, for example, one with which it has been combined. The relation information is intended to support the search for learning resources that are syntactically or semantically similar; it also ensures that when a learning resource is reauthored, it does not entirely lose the contextual information 
it has gathered to date.

Finally, the iLog system [13,9] tags learning objects with what it terms empirical usage metadata. The metadata is generated from observations about students' interactions with learning objects, for example how long they spend on a page or exercise, which is mined from session $\log$ files. Feature selection and data mining techniques are employed to isolate salient characteristics associated with particular learning outcomes, then, an association rule mining algorithm generates the resulting prediction rules. For example a rule might dictate that if a student has not yet taken a calculus course, and the student found the learning object confusing, then their likelihood of failure is $27 \%$. The empirical usage metadata is finally generated based on the salient characteristics and the extracted rules.

\subsection{Contextual Usage Metadata Federation}

As e-learning environments become more serviceoriented and incorporate multiple learning resource providers, the exchange of contextual usage metadata will become increasingly important. Sharing the metadata allows a more informed view of user interactions with each learning resource and provides benefits for both course authors and learners such as improved reuse and personalisation of learning resources.

Many of the previously mentioned approaches that facilitate usage metadata also incorporate methods to reconcile the usage metadata from various sources. In the work of Najjar et al., [10] the attention metadata approach uses the common open standard, AttentionXML, to track usage metadata across multiple systems. Usage metadata from log files is converted into the AttentionXML format using manually constructed transforms and the resulting data streams are merged.

In McCalla's work on the ecological approach to elearning environments [8], usage metadata is essentially derived from attaching user models to individual learning objects. To reuse the learning objects requires a means to provide interoperability of user models or, as suggested by McCalla, a standardised user model. He also suggests related work examining agent-based learning objects as a means to mediate interoperability of usage metadata.

The iLog approach presented by Riley et al. [13] uses learning object wrappers to transfer usage metadata to a server where it is processed and attached to a standardbased metadata model for that learning object.

The LIS.KOM framework proposed by Lehmann et al. [7] also uses a centralised server to process the usage metadata. Here, the usage metadata is provided by local clients that collect data from custom add-ins in systems such as learning management systems and repositories.

These are all interesting and varied approaches to usage metadata federation that provide different levels of integration. However, due to the lack of standards in this area many of these approaches rely on the use of commonly adopted, custom metadata formats and methods to obtain, represent and transfer usage metadata between different locations. To integrate new systems in these approaches, the same formats and techniques will need to be fully adopted and this will likely require significant integration effort and expertise.

Looking beyond the area of usage metadata, there are other interesting approaches to federation in related areas. In more general metadata exchange, Haslhofer [6] describes mapping approaches to share digital resource metadata. As new mappings can be constructed for any new type of metadata the mapping approach allows significant flexibility and extensibility. The mapping approach is common in many fields such as database, ontology and user model integration. It is the approach adopted in FUMES [15] to provide user model interoperability and, as described in the following section, is extended in FUMES2 to support learning resource usage metadata.

\section{Dynamic Federation of Contextual Usage Metadata}

In this section we propose an approach, shown in Figure 1 , to provide dynamic contextual usage metadata from multiple sources for the reuse of learning resources in an adaptive educational environment. In particular, this section will focus on the metadata federation component FUMES2.

The Federated Usage Metadata Exchange Service (FUMES2) allows the dynamic capture, exchange and update of usage metadata between multiple heterogeneous sources. FUMES2 is an advancement of previous research into learner model exchange called the Federated User Model Exchange Service (FUMES) [15].

FUMES2 provides dynamic usage metadata exchange between multiple service components. For example, Figure 1 shows FUMES2 gathering and integrating explicit and implicit usage metadata from a course author in the course author service and the learners in the adaptive education service. This usage metadata can be used to support subsequent course authors in selecting and reusing learning resources.

Similarly, FUMES2 can be used to retrieve usage metadata from multiple external sources like learning management systems, learning repositories and various educational services. In this case, valuable usage metadata can be retrieved to enhance the selection of learning resources and to overcome "cold-start" problems in adaptive course design.

However, due to the diverse nature of these various sources this metadata is often very heterogeneous in structure, syntax and semantics. The lack of usage metadata 


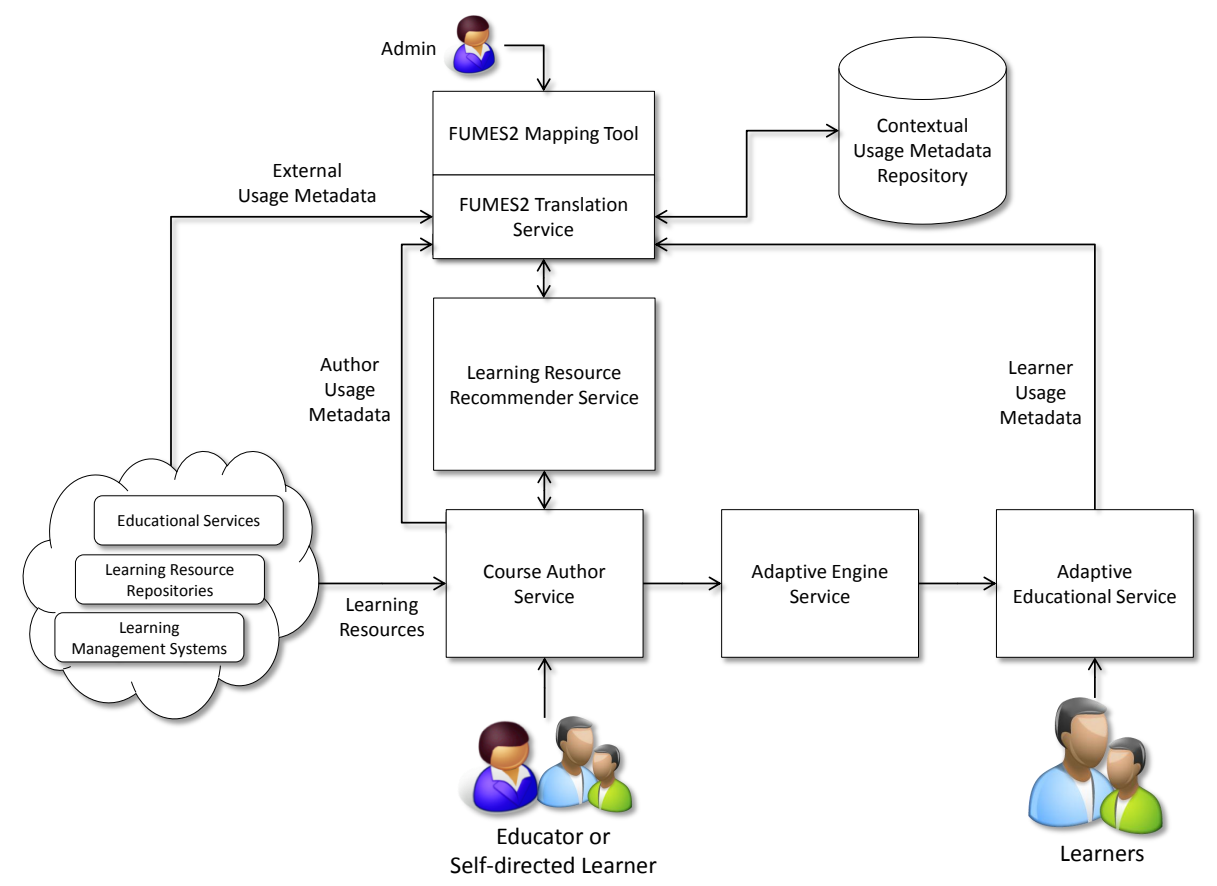

Figure 1. Learning Resource Reuse Approach

standardisation requires techniques to mediate this heterogeneity and in FUMES2 a mapping approach is adopted.

For sources that do not explicitly collect usage data for exchange purposes, a lightweight plug-in is used to filter and extract resource usage metadata from internal components such as $\log$ files. For example, in a learning management system detailed logs of user interactions with resources are usually recorded. These logs can be mined to provide detailed contextual resource usage metadata for many learners.

One of the major benefits of the FUMES2 mapping approach over the related work described in Section 2 is that the usage metadata does not have to conform to any particular standard or custom format and can be represented using the system's own structure and syntax. The mapping approach taken by FUMES2 allows greater flexibility in how the metadata in the various components is represented and reused. The usage metadata can be manipulated using various predefined mapping types available within FUMES2.

The FUMES2 mapping tool can be used to create mappings from the usage metadata of the various sources to a canonical usage metadata model. This canonical model encapsulates core types of usage metadata and is extensible to support varied types. The FUMES2 mapping tool is a web-based application that allows an administrator to rapidly create graphical mappings between equivalent usage metadata without the need to learn underlying transla- tion technologies.

Once the mappings have been created, they can be executed automatically and on-demand using the FUMES2 translation service. The translation service is a web service component that dynamically exchanges usage metadata between the various integrated services. When a service makes a request for updated usage metadata the translation service retrieves usage metadata from the other sources and executes the mappings previously created in the mapping tool. This performs a translation of the heterogeneous usage metadata into the common canonical model representation. Following this, a second set of mappings are executed to translate the canonical model into the target system's usage metadata representation.

Although it can be configured in different ways, in this scenario FUMES2 is used to translate usage metadata from multiple adaptive services and external systems into a centralised contextual usage metadata repository. This repository can be queried through FUMES2 and is the basis for the application of the learning resource recommender service. This service supplies recommendations for the reuse of learning resources to a course author, such as an educator or self-directing learner, in the course author service, as shown in Figure 1. The course author service allows the course author greater input and control of the resource selection process when facilitating the generation of personalised courses for learners. 
Implicit and explicit usage metadata from the course author service and adaptive educational service is fed back through FUMES2 to the contextual usage metadata repository thereby allowing a consistent feedback cycle for the update of resource usage metadata. This allows for more dynamic, up-to-date contextual usage metadata in this approach and ultimately allows more targeted personalisation for each individual learner.

\section{Contextual Usage Metadata for Reuse}

We have now an approach to federating contextual usage metadata from multiple heterogenous sources. We argue that such federation is necessary for compiling a collection of metadata large enough in order for meaningful patterns to be exposed. Certainly, gathering data from multiple sources alleviates somewhat the cold start situation where there is not contextual information about a particular learning resource yet in an individual system.

Clearly contextualised usage data is becoming a worthwhile consideration in e-learning systems. Related research has begun to explore how this information may be used to monitor not only when and how content is used, but also when and how (certain) services are used (contextualised attention metadata) and how learning resources are adapted (lifecycle information). Usage data supports both learner and educator in capitalising on the advantages of an online education environment.

However to date, learning resources have been seen as rather independent entities, where knowledge prerequisites are the only glue between different resources. In particular, the narratives of the courses, where the learning resources have been deployed has not yet been fully explored. We argue that such information is especially useful for scaffolding educators in designing quality e-learning courses.

Learning resources are not independent resources, and typically have many inherent links between them that go beyond the basic prerequisite knowledge. In fact learning resources are not always presented in sequence to the learner to begin with; this is especially true when the e-learning course contains both content and services. For example in a peer review exercise, the student might be presented with the content of the article under review, in parallel to services which allow him to rate and provide feedback on it.

Therefore, we believe that the contextual usage data that describes a resource should include details about the narrative of any course in which it has been used, i.e., the conceptual flow (which may be adaptive) which details the other learning resources contained in the course and the underlying structure between them. Of course, information describing the learners who have consumed the resources, how those resources were adapted or personalised, and any indications about the success of the resources deployment are still recorded.

The combination of this information can then be used to scaffold course authors. For example in Figure 2 the course author has some resources he wants to use in his course (A, $\mathrm{T}, \mathrm{J}, \mathrm{Q}, \mathrm{R}$ and $\mathrm{G}$ ) and is looking to add some more. The system can now recommend learning resources to the author that not only fulfil a particular pedagogic requirement, but that have been used in a similar context before. The author can examine whether that resource was successful, for which students or kinds of students it was particularly successful, and how it was adapted for those students.

\section{Summary}

Analysing how learning resources are used by others can greatly facilitate their reuse. In adaptive learning environments in particular, rich usage information can support course authors in the selection and reuse of appropriate learning resources. However, learning resources can be distributed in many heterogeneous locations making the gathering and exchanging of up-to-date usage data a difficult challenge. In this paper, we have discussed some related work in the area of usage metadata and its federation. Subsequently, we presented a new approach to facilitate the dynamic federation of usage metadata from multiple sources. This approach uses mapping techniques to provide a flexible and extensible method to overcome structural, syntactic and semantic heterogeneity in usage metadata. Following this, an approach is described that shows how federated usage metadata can be used to better facilitate the selection and reuse of learning resources.

\section{Acknowledgement}

This work was funded by Science Foundation Ireland via grant 08/IN.1/I2103: Adaptive and adaptable media and services for dynamic personalisation and contextualisation (AMAS).

\section{References}

[1] C. Brooks and G. McCalla. Towards flexible learning object metadata. Engineering Education, 16(1 - 2):50-63, 2006.

[2] P. Brusilovsky. Methods and techniques of adaptive hypermedia. User Modeling and User-Adapted Interaction, 6(23):87-129, July 1996.

[3] O. Conlan and V. Wade. Evaluation of APeLS - An Adaptive eLearning Service Based on the Multi-Model, MetadataDriven Approach. In P. De Bra and W. Nejdl, editors, Third International Conference on Adaptive Hypermedia and Adaptive Web-Based Systems (AH2004), pages 291295. Springer Berlin / Heidelberg, 2004. 


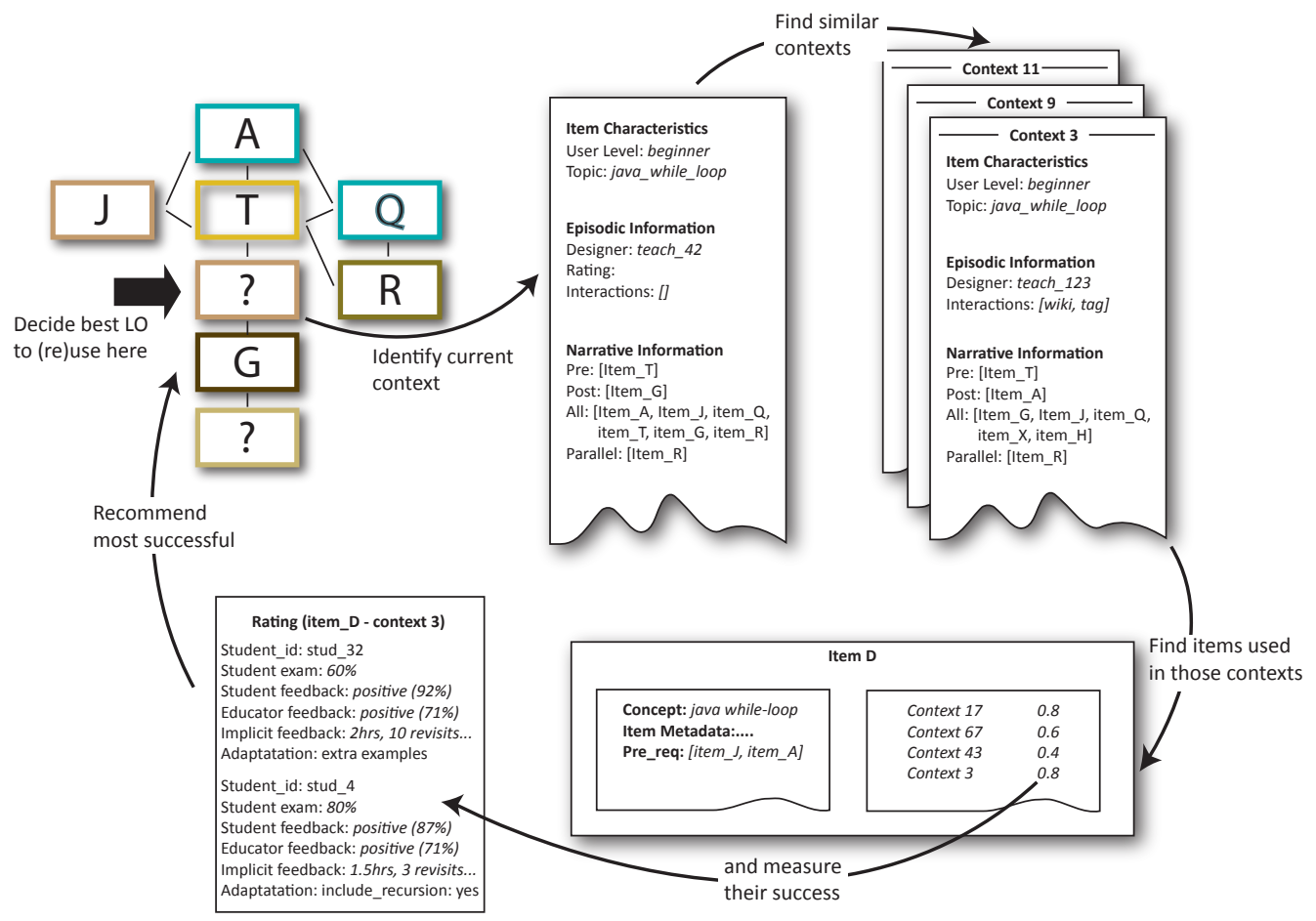

Figure 2. Strategy for Identifying Suitable LOs for (Re)Use

[4] D. Dagger, O. Conlan, and V. Wade. Personalisation for All: Making Adaptive Course Composition Easy. Special issue of the IEEE Journal of Educational Technology and Society, 8(3):9-25, July 2005.

[5] P. de Bra, D. Smits, and N. Stash. The Design of AHA! In Proceedings of the 17th ACM Conference on Hypertext and Hypermedia, pages 133 - 134, Odense, Denmark, Aug. 2006.

[6] B. Haslhofer and W. Klas. A survey of techniques for achieving metadata interoperability. ACM Computing Surveys, 42(2):1-37, 2010.

[7] L. Lehmann, T. Hildebrandt, C. Rensing, and R. Steinmetz. Capture, Management, and Utilization of Lifecycle Information for Learning Resources. IEEE Transactions on Learning Technologies, 1(1):75-87, Jan. 2008.

[8] G. McCalla. The Ecological Approach to the Design of ELearning Environments: Purpose-based Capture and Use of Information about Learners. Journal of Interactive Media in Education - Special Issue on the Educational Semantic Web, 7, 2004

[9] L. D. Miller, L.-K. Soh, B. Neilsen, K. Kupzyk, A. Samal, E. Lam, and G. Nugent. Revising computer science learning objects from learner interaction data. In Proceedings of the 42nd ACM technical symposium on Computer science education - SIGCSE '11, pages 45 - 50, Dallas, Texas, USA, Mar. 2011. ACM Press.

[10] J. Najjar, E. Duval, and M. Wolpers. Attention Metadata: Collection and Management. In In Proceedings of the workshop on logging traces of web activity: the mechanics of data collection, at the 15th International World Wide Web
Conference ( $W W W$ 06), pages 1 - 4, Edinburgh, Scotland, May 2006.

[11] X. Ochoa and E. Duval. Use of contextualized attention metadata for ranking and recommending learning objects. In Proceedings of the 1st international workshop on Contextualized attention metadata: collecting, managing and exploiting of rich usage information (CAMA 06), pages 9 - 16, Arlington, VA, USA, Nov. 2006.

[12] I. O'Keeffe and V. Wade. Personalised Web Experiences: Seamless Adaptivity across Web Service Composition and Web Content. In User Modeling, Adaptation and Personalization, pages 480-485. Springer Berlin / Heidelberg, 2009.

[13] S. Riley, L. Miller, L.-K. Soh, A. Samal, and G. Nugent. Intelligent Learning Object Guide (iLOG): A Framework for Automatic Empirically-Based Metadata Generation. In Proceeding of the 2009 conference on Artificial Intelligence in Education, pages 515-522, Brighton, UK, July 2009.

[14] V. P. Wade and H. Ashman. Guest Editors' Introduction: Evolving the Infrastructure for Technology-Enhanced Distance Learning. IEEE Internet Computing, 11(3):16-18, May 2007.

[15] E. Walsh, A. O'Connor, and V. Wade. Supporting Learner Model Exchange in Educational Web Systems. In 7th International Conference on Web Information Systems and Technologies, Noordwijkerhout, The Netherlands, May 2011.

[16] M. Wolpers, J. Najjar, K. Verbert, and E. Duval. Tracking Actual Usage: the Attention Metadata Approach. Educational Technology \& Society, 10(3):106 - 121, 2007. 